\title{
Comparaison de deux diagonales pour un ensemble bisimplicial
}

\author{
Michel Zisman
}

Received: 22 October 2014 / Accepted: 16 November 2014 / Published online: 2 December 2014

(C) Tbilisi Centre for Mathematical Sciences 2014

\begin{abstract}
In this paper a proof is given that the two diagonals of any bisimplicial set, by Quillen and Artin-Mazur respectively, are weakly homotopy equivalent.
\end{abstract}

Keywords Bisimplicial set · Diagonal · Homotopy type

\section{Introduction}

En 1965, dans le même numéro de Topology Artin et Mazur [1] d'une part et Quillen [2] de l'autre définissent chacuns une notion de diagonale pour un ensemble bisimplicial $X$. On désigne par $D$ celle d'Artin-Mazur et par $\nabla$ celle de Quillen. Alors $D(X)_{n} \subset$ $\prod_{p+q=n} X_{p, q}$ et $\nabla(X)_{n}=X_{n, n}$. Soit $\varphi: \nabla \rightarrow D$ le morphisme fonctoriel défini $\operatorname{par} \varphi_{n}(X)(x)=\left\{d_{p+1}^{\prime} \cdots d_{n}^{\prime} d_{0}^{\prime \prime p} x\right\}_{p+q=n}$ pour $x \in X_{n, n}$. Le but de cet article est de démontrer le théorème suivant. ${ }^{1}$

Théorème $\varphi$ est une équivalence d'homotopie (faible).

Dans la suite on écrira simplement "équivalence d'homotopie". En particulier si $\Gamma$ est un monoïde simplicial et si $X$ est l'ensemble bisimplicial $X_{p, q}=\left(\Gamma_{q}\right)^{p}$ avec les opérateurs faces et dégénérescences usuels, $D(X)$ n'est autre que $\bar{W}(\Gamma)$, la barconstruction de $\Gamma$, qui a donc même type d'homotopie que $\nabla(X)$, ce qui est d'ailleurs

\footnotetext{
1 Ce texte est une version remaniée en 1990 d'un manuscrit de 1987 destiné à Jean-Marc Cordier [4] qui avait besoin de ce résultat pour terminer sa thèse. La première démonstration publiée du théorème se trouve dans l'article de Cegarra-Remedios de 2005 [3]. Depuis 2005, d'autres démonstrations ont parues. Je remercie Antonio Martinez Cegarra qui m'a incité à publier celle-ci.
}

Communicated by Antonio Cegarra.

M. Zisman $(\bigotimes)$

Université Paris 7, Paris, France

e-mail: mzisman28@gmail.com 
facile à démontrer directement. Si $X$ est un groupe abélien bisimplicial, on retrouve le théorème d'Eilenberg-Zilber-Cartier.

\section{Quelques foncteurs}

2.1 Soit $\mathcal{S}$ la catégorie des ensembles simpliciaux, et soit $\mathcal{S}^{2}$ celle des ensembles bisimpliciaux. Les deux constructions suivantes sont bien connues:

Notons $\Delta[p, q]$ l'ensemble bisimplicial, donc le foncteur contravariant de $\Delta \times$ $\Delta$ dans Ens, défini par $\Delta(?, p) \times \Delta(?, q)$. Soit $L: \Delta \rightarrow \mathcal{S}^{2}$ le foncteur “diagonale" $[n] \mapsto \Delta[n, n]$.

Introduisons maintenant la "moitiê" $\Lambda$ du foncteur $L$; plus précisément, soit $\Lambda[n]_{p, q}$ l'ensemble des couples $(\alpha, \beta)$ de fonctions croissantes $\alpha:[p] \rightarrow[n], \beta:[q] \rightarrow[n]$ telles que pour tout $u \in[p]$ et tout $v \in[q]$, on a $\alpha(u) \leq \beta(v)$. On définit bien ainsi un sous-ensemble bisimplicial $\Lambda[n]$ de $\Delta[n, n]$. Mieux, la correspondance $[n] \mapsto \Lambda[n]$ définit un foncteur $\Delta \rightarrow \mathcal{S}^{2}$ qui est un sous-foncteur du foncteur $L:[n] \mapsto \Delta[n, n]$.

Lemme $1 \Lambda[n]$ est la colimite du diagramme

$$
\begin{aligned}
\Delta[0, n] \stackrel{\Delta\left[i d, \partial^{0}\right]}{\longleftarrow} \Delta[0, n-1] \stackrel{\Delta\left[\partial^{1}, i d\right]}{\longrightarrow} \Delta[1, n-1] \stackrel{\Delta\left[i d, \partial^{0}\right]}{\longleftarrow} \Delta[1, n-2] \stackrel{\left.\Delta\left[\partial^{2}, i d\right]\right]}{\longrightarrow} \\
\Delta[2, n-2] \longleftarrow \stackrel{\Delta\left[i d, \partial^{0}\right]}{\longleftarrow} \Delta[n-1,0] \stackrel{\Delta\left[\partial^{n}, i d\right]}{\longrightarrow} \Delta[n, 0] .
\end{aligned}
$$

Démonstration On commence par donner la définition équivalente suivante du foncteur $\Lambda$. Soit $\alpha \times \beta:[p] \times[q] \rightarrow[n] \times[n]$ un élément de $\Lambda[n]_{p, q}$. On lui fait correspondre de façon biunivoque le triplet $p, q, \gamma_{p, q} \in \Delta[n]_{p+q+1},\left(\gamma_{p, q}\right.$ est donc une fonction croissante $[p+q+1] \rightarrow[n])$, en posant

$$
\gamma_{p, q}(i)= \begin{cases}\alpha(i) & \text { pour } 0 \leq i \leq p \\ \beta(i-p-1) & \text { pour } \quad p+1 \leq i \leq p+q+1\end{cases}
$$

Soit $a \times b:[p] \times[q] \rightarrow\left[p^{\prime}\right] \times\left[q^{\prime}\right]$ un morphisme de $\Delta \times \Delta$, et soit $\alpha^{\prime} \times \beta^{\prime} \in \Lambda[n]_{p^{\prime}, q^{\prime}}$. On a $\Lambda[n]_{a, b}\left(\alpha^{\prime} \times \beta^{\prime}\right)=\left(\alpha^{\prime} \circ a\right) \times\left(\beta^{\prime} \circ b\right)$, et soit $\gamma_{p, q}$ l'élément de $\Delta_{p+q+1}[n]$ qui lui correspond ; si à $\alpha^{\prime} \times \beta^{\prime}$ correspond $\gamma_{p^{\prime}, q^{\prime}}^{\prime} \in \Delta_{p^{\prime}+q^{\prime}+1}[n]$, on a

$$
\gamma_{p, q}(i)=\left\{\begin{array}{lll}
\gamma_{p^{\prime}, q^{\prime}}^{\prime} \circ a(i) & \text { pour } 0 \leq i \leq p \\
\gamma_{p^{\prime}, q^{\prime}}^{\prime} \circ b(i-p-1)+p^{\prime}+1 & \text { pour } & p+1 \leq i \leq p+q+1
\end{array}\right.
$$

En particulier, pour $p+q=n$, notons $\omega_{p, q}:[n+1] \rightarrow[n]$ l'application $k \mapsto k$ si $k \leq p$ et $k \mapsto k-1$ sinon. Il résulte des formules précédentes que l'on a

$$
d_{p}^{\prime} \omega_{p, q}=d_{0}^{\prime \prime} \omega_{p-1, q+1}
$$


Si maintenant $(\alpha, \beta)$ est un élément quelconque de $\Lambda[n]_{u, v}$, et si $p$ est un entier vérifiant $\alpha(u) \leq p \leq \beta(0)$, on définit $a_{p}:[u] \rightarrow[p]$ et $b_{q}:[v] \rightarrow[p]$ en posant $a_{p}(k)=\alpha(k)$ et $b_{q}(k)=\beta(k)-p$, si bien que l'on a $\Lambda[n]_{a, b}\left(\omega_{p, q}\right)=(\alpha, \beta)$. Enfin si on a $\alpha(u) \leq p<p+1 \leq \beta(0)$ on a

$$
a_{p+1}=\partial^{p+1} \circ a \text { et } b_{q}=\partial^{0} \circ b_{q-1} .
$$

La démonstration du lemme résulte maintenant aisément de ces résultats explicites. Soit $\lambda: \Lambda[n] \rightarrow X$ un morphisme de $\mathcal{S}$. On pose, (pour $p+q=n), \lambda\left(\omega_{p, q}\right)=x_{p, q}$ Ces éléments vérifient les égalités $d_{p}^{\prime} x_{p, q}=d_{0}^{\prime \prime} x_{p-1, q+1}$ qui résultent des formules analogues pour les $\omega$. Réciproquement, si la famille des $\left(x_{p, q} \in X_{p, q}\right)$ vérifie les égalités précédentes, on lui fait correspondre un unique élément $\lambda \in \Lambda[n]$, en posant, avec les notations définies plus haut, $\lambda(\alpha, \beta)=X_{a_{p}, b_{q}}\left(x_{p, q}\right)$. Cette définition est indépendante du choix de $p$.

2.2 Foncteurs adjoints. Rappelons qu'à la donnée d'un foncteur $f: \mathcal{C} \rightarrow \mathcal{E}$, où $\mathcal{E}$ est une catégorie possédant des colimites, sont associés deux foncteurs adjoints $g: \mathcal{C}^{\text {opp }} E n s \rightarrow \mathcal{E}$ adjoint à gauche de $r: \mathcal{E} \rightarrow \mathcal{C}^{\text {opp }} E n s$, et définis comme suit. Pour tout objet $e$ de $\mathcal{E}$, on a $r(e)=\mathcal{E}(f(?), e)$; si $X=\mathcal{C}(?, c)$ où $c$ est un objet de $\mathcal{C}$, alors on pose $g(X)=f(c)$, et dans le cas général, pour tout objet $X$ de $\mathcal{C}^{\text {opp }} E n s$, on a $g(X)=$ colim $f(c)_{x}$, où $f(c)_{x}=f(c)$, où $c$ parcourt les objets de $\mathcal{C}$ et où enfin la colimite est prise sur tous les morphismes $\mathcal{C}($ ?,$c) \rightarrow X$.

Appliquons ces constructions aux deux foncteurs $\Lambda$ et $L$ de $\Delta$ dans $\mathcal{S}^{2}$ qui définissent donc respectivement des foncteurs $U$ et $V: \mathcal{S} \rightarrow \mathcal{S}^{2}$ adjoints à gauche des foncteurs $D$ et $\nabla: \mathcal{S}^{2} \rightarrow \mathcal{S}$. Il est clair sur les définitions que $\nabla$ est bien le foncteur diagonal de Quillen, et le Lemme 1 exprime le fait que $D$ est la diagonale définie par Artin-Mazur. Par ailleurs, l'inclusion $\Lambda \hookrightarrow L$ induit un morphisme fonctoriel $\varphi: \nabla \rightarrow D$ qui coïncide avec celui défini dans l'introduction. Enfin $\nabla$ possède aussi un adjoint à droite $P: \mathcal{S} \rightarrow \mathcal{S}^{2}$ défini par $P(Y)=\mathcal{S}(\Delta[?] \times \Delta[?], Y)$.

Voici quelques exemples. Soient $X$ et $Y$ deux ensemble simpliciaux, et notons $X \otimes Y$ l'ensemble bisimplicial $(X \otimes Y)_{p, q}=X_{p} \times Y_{q}$. En particulier, il vient $\Delta[p, q]=$ $\Delta[p] \otimes \Delta[q]$. On montre facilement que l'on a $\nabla(X \otimes Y)=D(X \otimes Y)=X \times Y$ en particulier $\nabla(\Delta[n, n])=D(\Delta[n, n])=\Delta[n] \times \Delta[n]$. Par ailleurs $\nabla(\Lambda[n])$ est la "moitié" de $\Delta[n] \times \Delta[n]$ i.e., en degré $p$ c'est l'ensemble des couples $(\alpha, \beta)$ de morphismes $[p] \rightarrow[n]$ tels que $\alpha(p) \leq \beta(0)$.

Déterminons enfin $D \Lambda[n]$. En degré p, c'est $\mathcal{S}^{2}(\Lambda[p], \Lambda[n])$. Soient $a$ et $b$ deux morphismes $[p] \rightarrow[n]$ tels que, pour tout $0 \leq k \leq p$ on a $a(k) \leq b(k)$. On leur associe un $\lambda: \Lambda[p] \rightarrow \Lambda[n]$ en posant $\lambda(\alpha, \beta)=(a \circ \alpha, b \circ \beta) \operatorname{pour}(\alpha, \beta) \in \Lambda[p]_{u, v}$. Ce morphisme est lui même déterminé, d'après la démonstration du Lemme 1, par la famille $x_{u, v}=\left(\theta_{u}, \tau_{v}\right) \in \Lambda[n]_{u, v}$ avec $u+v=p$, où l'on a posé $\theta_{u}(i)=a(i)$ pour $i \in[u]$ et $\tau_{v}(j)=b(p-v+j)$ pour $j \in[v]$.

Réciproquement, se donner un morphisme $\Lambda[p] \rightarrow \Lambda[n]$ c'est se donner une famille $\left(x_{u, v}=\left(\theta_{u}, \tau_{v}\right) \in \Lambda[n]_{u, v}\right)$ avec $u+v=p$ satisfaisant à certaines conditions. Posons, pour $0 \leq k \leq p, a(k)=\theta_{k}(k), b(k)=\tau_{p-k}(0)$. Les relations qui lient les $x_{u, v}$ montrent que les deux fonctions ainsi définies sont croissantes; par ailleurs on a 
$a(k) \leq b(k)$. Finalement, on a les inclusions

$$
\nabla \Lambda[n] \subset D \Lambda[n] \subset D L[n]=\Delta[n] \times \Delta[n]
$$

Le foncteur $k: \Delta \times \Delta \rightarrow \mathcal{S}$. Il est défini sur les objets par $k([p] \times[q])=\Delta[p+q+1]$ et sur les flèches $a \times b:[p] \times[q] \rightarrow\left[p^{\prime}\right] \times\left[q^{\prime}\right]$ par

$$
k(a, b)(i)= \begin{cases}a(i) & \text { pour } 0 \leq i \leq p \\ b(i-p-1)+p^{\prime}+1 & \text { pour } p+1 \leq i \leq p+q+1 .\end{cases}
$$

Partant de la catégorie $\mathcal{C}=\Delta \times \Delta$, le foncteur $k$ permet de définir deux foncteurs adjoints reliant $\mathcal{S}$ et $\mathcal{S}^{2}$. Soit $G$ l'adjoint à gauche et appelons provisoirement $\mathcal{D}$ l'adjoint à droite.

Lemme 2 Lefoncteur $\mathcal{D}$ est canoniquement isomorphe à $U$. En particulier, $U$ possède un adjoint à gauche et un adjoint à droite.

Démonstration Par définition, on a

$$
\mathcal{D}(X)_{p, q}=\mathcal{S}(\Delta[p+q+1], X)=X_{p+q+1},
$$

et en particulier si on prend $X=\Delta[n]$, il résulte de formules données dans la démonstration du Lemme 1, que l'on a

$$
\mathcal{D}(\Delta[n])=\Lambda[n]=U(\Delta[n]),
$$

et de la formule $(a)$ que $\mathcal{D}$ commute aux colimites. D'où le résultat.

Dans la suite, on identifiera donc les deux foncteurs précédents. Mais alors pour tout ensemble simplicial $Y$ il vient $\nabla U(Y)_{n}=Y_{2 n+1}$, et, pour tout $y \in Y_{2 n+1}$ considéré comme élément de $\nabla U(Y)_{n}$, il vient

$$
\begin{gathered}
\bar{d}_{i}=d_{i} d_{n+1+i} y=d_{n+i} d_{i} y \quad \text { pour } \quad o \leq i \leq n \\
\bar{s}_{i}=s_{i} s_{n+1+i} y=s_{n+i} s_{i} y \text { pour } \quad o \leq i \leq n
\end{gathered}
$$

où faces et dégénérescences de $\nabla U(Y)$ ont été surlignées pour les distinguer de celles de $Y$.

On définit un morphisme fonctoriel $i:$ Id $\rightarrow \nabla U$ par ses valeurs sur les $\Delta[n]$ (car les deux membres commutent aux colimites ), i.e. par des applications "fonctorielles" en $n i_{\Delta[n]}: \Delta[n] \rightarrow \nabla U(\Delta[n])$, c'est-à-dire par des éléments de $\Delta[n]_{2 n+1}$, soit finalement par des fonctions croissantes $i_{n}:[2 n+1] \rightarrow[n]$. On choisit $i_{n}(k)=k$ pour $k \leq n-1$ et $i_{n}(k)=n$ sinon.

Pour en terminer avec ces préliminaires, il reste à expliciter $G$ et $D U$.

Explicitation de $G$ et $D U$. Appliquons le foncteur $G$ au diagramme (*) : puisque par définition, $G(\Delta[p, q])$ est égal à $\Delta[p+q+1]$, c'est que $G(\Lambda[p])$ est la colimite du diagramme 


$$
\Delta[p+1] \stackrel{\Delta\left[\partial^{1}\right]}{\longleftarrow} \Delta[p] \stackrel{\Delta\left[\partial^{1}\right]}{\longrightarrow} \Delta[p+1] \stackrel{\Delta\left[\partial^{2}\right]}{\longleftarrow} \Delta[p] \stackrel{\Delta\left[\partial^{2}\right]}{\longrightarrow} \Delta[p+1] \cdots \stackrel{\Delta\left[\partial^{p}\right]}{\longrightarrow} \Delta[p],
$$

c'est-à-dire $\Delta[1] \times \Delta[p]$. Mais alors il vient, pour l'ensemble simplicial $X$, $D U(X)_{p}=\mathcal{S}(\Delta[p], D U(X))=\mathcal{S}^{2}(U(\Delta[p]), U(X))=\mathcal{S}^{2}(\Lambda[p], U(X))=$ $\mathcal{S}(G(\Lambda[p]), X)=\mathcal{S}(\Delta[1] \times \Delta[p], X)=\mathcal{H}$ om $(\Delta[1], X)_{p}$. Ainsi finalement on a $D U(X)=\mathcal{H} \operatorname{om}(\Delta[1], X)$ : c'est l'espace des chemins (libres) de $X$.

Le diagramme (*) permet facilement de montrer aussi que le morphisme d'adjonction $G U(\Delta[n]) \rightarrow \Delta[n]$ est simplement la projection $\Delta[1] \times \Delta[n] \rightarrow \Delta[n]$. Donc le morphisme d'adjonction $G U(X) \rightarrow X$ est la projection $\Delta[1] \times X \rightarrow X$ car $G U$ commute aux colimites. Mais alors pour tout objet $Z$ de $\mathcal{S}$ le morphisme d'adjonction $j_{Z}: Z \rightarrow D U(Z)$ est l'application $\mathcal{H o m}(\Delta[0], Z) \rightarrow \mathcal{H}$ om $(\Delta[1], Z)$ induite par l'unique application $\Delta[1] \rightarrow \Delta[0]$ (unicité de l'adjoint à droite du foncteur $G U=? \times \Delta[1])$.

\section{Démonstration du théorème}

3.1 Commençons par étudier le morphisme fonctoriel $i: \operatorname{Id} \rightarrow \nabla U$.

Proposition 1 i est une équivalence d'homotopie.

Démonstration c'est une conséquence des deux lemmes suivants.

Lemme 3 Le foncteur $\nabla U$ possède les trois propriétés suivantes:

i) il commute aux colimites

ii) il transforme monomorphismes en monomorphismes

iii) $\nabla U(\Delta[n])$ est contractile.

Lemme 4 Si un foncteur $F: \mathcal{S} \rightarrow \mathcal{S}$, muni d'un morphisme fonctoriel $\rho:$ id $\rightarrow F$, vérifie les conditions i), ii) et iii) du lemme précédent, alors $\rho$ est une équivalence d'homotopie.

Démonstration du lemme 3 Les deux premières propriétés proviennent du fait que ce foncteur possède un adjoint à droite et un adjoint à gauche. Posons $F=\nabla U$ et montrons plus généralement que $F$ transforme des applications $f, g: X \rightarrow Y$ homotopes (rel points bases) en des applications homotopes (rel points bases). Cela résulte du fait que $F$ commute aux produits et du fait que $F(\Delta[1])$ n'est autre que la réunion de deux exemplaires de $\Delta[1]$ mis bout-à bout:

$$
\nabla \Lambda[1]=\nabla U(\Delta[1]): \quad 0 \stackrel{\Delta[1]}{\longrightarrow} 1 \stackrel{\Delta[1]}{\longrightarrow} 2 .
$$

Le complément concernant les points bases vient de l'égalité $F(\Delta[0])=\Delta[0]$.

Démonstration du lemme 4 Elle utilise le résultat technique suivant, dont on peut aussi énoncer une version pointée: soient $\mathcal{C}$ et $\mathcal{C}^{\prime}$ deux carrés cocartésiens de la catégorie $\mathcal{S}$ 

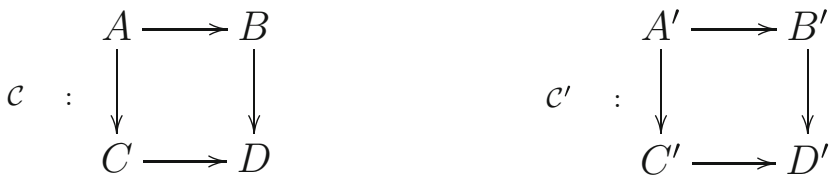

où les flèches verticales sont des monomorphismes, et soit $f=\left(f_{A}, f_{B}, f_{C}, f_{D}\right)$ : $\mathcal{C} \rightarrow \mathcal{C}^{\prime}$ un morphisme. Alors si $f_{A}, f_{B}$ et $f_{C}$ sont des équivalences d'homotopies, il en va de même de $f_{D}$ (Lemme d'amalgamation).

Introduisons quelques notations. D'abord soit $\mathrm{Sk}^{n} X$ le $n$-squelette de l'ensemble simplicial $X$; notons $\dot{\Delta}[n]=\mathrm{Sk}^{\mathrm{n}-1} \Delta[\mathrm{n}]$ la $(n-1)$-sphère bord de $\Delta[n]$, et montrons par récurrence sur $n$ que $\rho_{\mathrm{Sk}^{n} X}$ est une équivalence d'homotopie pour tout objet $X$ de $\mathcal{S}$. Pour $n=0$ on a

$$
F\left(\mathrm{Sk}^{0}\right)=F\left(\coprod_{x \in X_{0}} \Delta[0]_{x}\right)=\coprod_{x \in X_{0}} F(\Delta[0])_{x}
$$

car $F$ commute aux colimites. Le résultat s'ensuit, d'après la condition iii). Soit maintenant $n \geq 2$ et considérons le carré cocartésien $\mathcal{C}$ dont les flèches verticales sont injectives:

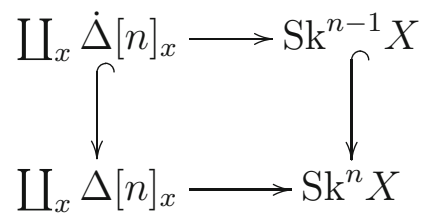

où $x$ parcourt l'ensemble des $n$-simplexes non dégénérés de $X$. Appliquons lui le foncteur $F$, il vient un carré cocartésien $\mathcal{C}^{\prime}$ dont les flèches verticales sont encore des monomorphismes:

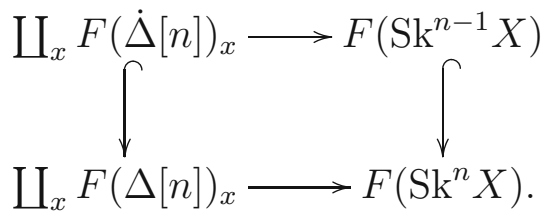

Prenons pour morphisme $f: \mathcal{C} \rightarrow \mathcal{C}^{\prime}$, le morphisme induit par le morphisme fonctoriel $\rho$. Comme $\rho_{\Delta[n]}$ est une équivalence d'homotopie d'après iii), et que $\rho_{\dot{\Delta}[n]}$ et $\rho_{\mathrm{Sk}^{n-1}(X)}$ sont des équivalences d'homotopie d'après l'hypothèse de récurrence, il résulte du résultat technique que c'est aussi vrai pour $\rho_{\mathrm{Sk}^{n}(X)}$.

Pour terminer la démonstration, il suffit de prendre la colimite des lignes du diagramme dont toutes les flèches verticales, induites par $\rho$ sont des équivalences d'homotopies: 


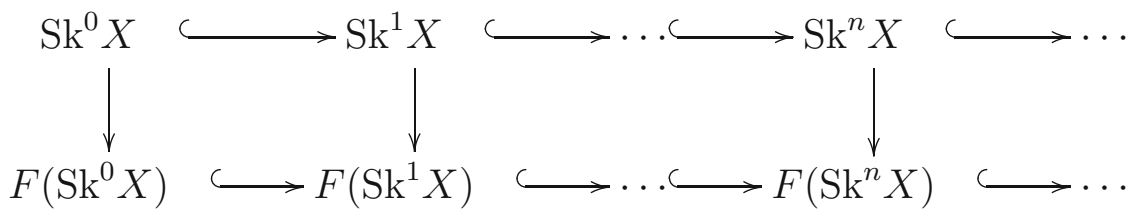

et donc, à la limite, $\rho_{X}$ aussi.

3.2 Un diagramme commutatif. La flèche $\Delta[0] \stackrel{\Delta\left[\partial^{1}\right]}{\rightarrow} \Delta[1]$ induit une application $\lambda_{X}: \mathcal{H o m}(\Delta[1], X) \rightarrow \mathcal{H} \operatorname{om}(\Delta[0], X)$ qui est une homotopie équivalence (utiliser le morphisme $\Delta[1] \times \Delta[1] \rightarrow \Delta[1]$ défini par l'application croissante $h:[1] \times[1] \rightarrow$ [1], telle que $(0,0),(1,0),(0,1)$ vont sur 0 et $(1,1)$ sur 1$)$. Puisque l'on a $\lambda_{X} \circ j_{X}=i d$, $j_{X}$ est aussi une équivalence d'homotopie.

La flèche $[p] \stackrel{u_{p}}{\rightarrow}[2 p+1]$, avec $u_{p}=\partial^{2 p+1} \circ \partial^{2 p} \circ \cdots \circ \partial^{p+1}$, induit une application $X_{2 p+1} \rightarrow X_{p}$ et, mieux, un morphisme $\rho_{X}: \nabla U(X) \rightarrow X$.

Lemme 5 Le diagramme

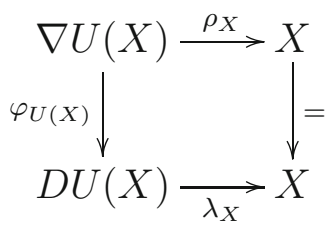

est commutatif.

Démonstration En effet, par adjonction, et compte tenu des explicitations précédentes, on se ramène à la commutativité du diagramme

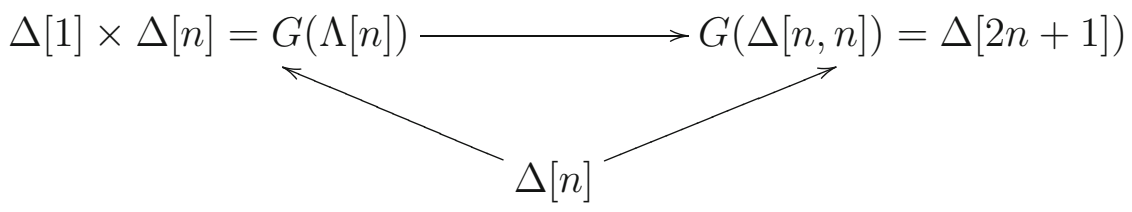

dont la flèche horizontale provient de $(0, i) \mapsto i,(1, i) \mapsto n+i+1$, la flèche oblique de droite de $i \mapsto(0, i)$ et celle de droite de $i \mapsto i$ pour $0 \leq i \leq n$.

Comme on a $\rho_{X} \circ i_{X}=\operatorname{Id} d_{X}$ et que d'après la Proposition $1, i_{X}$ est une équivalence, il en va de même pour $\rho_{X}$, et le diagramme commutatif précédent montre que le théorème est vrai pour les ensembles bisimpliciaux de la forme $U(Y)$.

Il reste à ramener le cas général à celui-ci.

3.3 Fin de la démonstration. Soit $\tilde{\varphi}_{X}: U \nabla(X) \rightarrow X$ l'adjoint de $\varphi_{X}$ : $\nabla(X) \rightarrow D(X)$, qui s'explicite comme suit: si $x_{p, q}$ est un élément de $U \nabla(X)_{p, q}=$ $X_{p+q+1, p+q+1}$, on a

$$
\tilde{\varphi}\left(x_{p, q}\right)=d_{p+1}^{\prime} \ldots d_{p+q+1}^{\prime} d_{0}^{\prime \prime p+1} x_{p, q} .
$$


Mais alors, $\nabla \tilde{\varphi}_{X}: \nabla U \nabla(X) \rightarrow \nabla(X)$ est, en degré $n$, l'application $X_{2 n+1,2 n+1} \rightarrow$ $X_{n, n}$ donnée par $x \mapsto d_{n+1}^{\prime} \cdots d_{2 n+1}^{\prime} d_{0}^{\prime \prime n+1} x$.

Par ailleurs, $\rho_{\nabla(X)}: \nabla U \nabla(X) \rightarrow \nabla(X)$ est donnée par $x \mapsto d_{n+1}^{\prime} \ldots$ $d_{2 n+1}^{\prime} d_{n+1}^{\prime \prime} \ldots d_{2 n+1}^{\prime \prime} x$. Ces deux applications sont homotopes, une homotopie étant donnée par les

$$
k_{i}(x)=s_{i}^{\prime} d_{n+1}^{\prime} \ldots d_{2 n+1}^{\prime} d_{i+1}^{\prime \prime} \ldots d_{i+n}^{\prime \prime} x
$$

Soit $\psi: U D \rightarrow$ id le morphisme d'adjonction. Le composé $\psi_{X} \circ U\left(\varphi_{X}\right)$ n'est autre que $\tilde{\varphi}_{X}$. En appliquant $\nabla$ à cette égalité, il vient $\nabla \psi_{X} \circ \nabla U \varphi_{X}=\nabla \tilde{\varphi}_{X}$. Par ailleurs le composé

$$
D(X) \stackrel{j_{D(X)}}{\longrightarrow} D U D(X) \stackrel{D \psi_{X}}{\longrightarrow} D(X)
$$

est l'identité, et nous savons déjà que $j_{D(X)}$ est une équivalence d'homotopie, donc aussi $D\left(\psi_{X}\right)$. Nous pouvons résumer ces résultats dans le diagramme ci-dessous

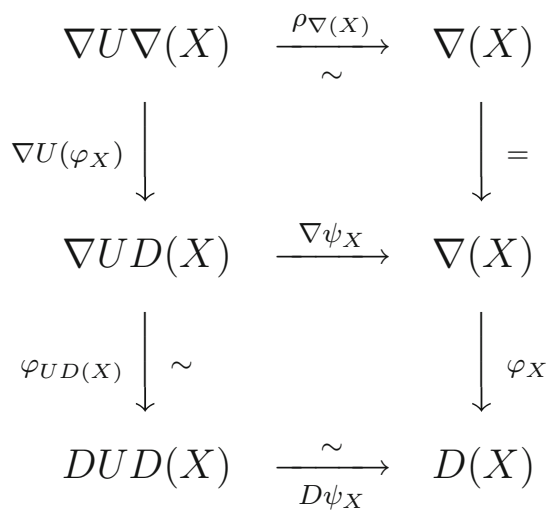

dont le carré du haut est homotopiquement commutatif, et celui du bas est commutatif, et où les flèches marquées d'un sont des équivalences d'homotopie.

Plaçons nous maintenant dans la catégorie homotopique où ces équivalences d'homotopie deviennent des isomorphismes, et les applications homotopes deviennent égales. Nous avons un diagramme commutatif

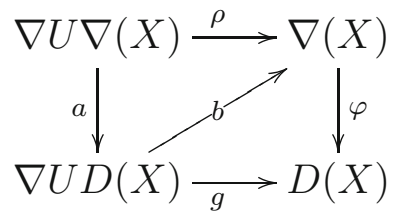

dans lequel $\rho$ et $g$ sont des isomorphismes. Mais alors, $\varphi$ est un isomorphisme d'inverse $b g^{-1}$, ce qui achève la démonstration. 


\section{References}

1. Artin, M., Mazur, B.: On the Van Kampen theorem. Topology 5, 179-189 (1966)

2. Quillen, D.: Spectral sequences of a double semi-simplicial group. Topology 5, 155-157 (1966)

3. Cegarra, A.M., Remedios, J.: The relationship between the diagonal and the bar-constructions on a bisimplicial set. Topol. Appl. 153, 21-51 (2005)

4. Cordier, J.-M.: Sur les limites homotopiques de diagrammes homotopiquement cohérents. Compositio Math. 62, 367-388 (1987) 